
$\angle$ Research Square
Preprints are preliminary reports that have not undergone peer review.
They should not be considered conclusive, used to inform clinical practice, or referenced by the media as validated information.

\title{
Occurrence and health risk assessment of perfluorinated substances from water in residential areas around fluorine chemical industry, China
}

\author{
Jiaxi Tang \\ Liaoning Technical University \\ Yongle Zhu \\ Liaoning Technical University \\ Yu Li \\ Liaoning Technical University

\section{Biao Xiang} \\ Liaoning Technical University \\ Ting Tan \\ Liaoning Technical University \\ Linyou Lv \\ Liaoning Academy of Agricultural Sciences \\ Qing Luo ( $\square$ luoqingyt@126.com ) \\ Shenyang University
}

\section{Research Article}

Keywords: Per- and polyfluoroalkyl substances, perfluorooctanoic acid, concentration, distribution, risk quotient, health risk assessment

Posted Date: August 10th, 2021

DOI: https://doi.org/10.21203/rs.3.rs-749717/v1

License: (a) (i) This work is licensed under a Creative Commons Attribution 4.0 International License. Read Full License 


\section{Abstract}

To identify the contamination status and assess the health risk of per-and polyfluoroalkyl substances (PFASs) in surface and ground water are of great significance recently. Eighteen individual PFASs were analyzed in thirty-three surface/ground water samples from fluorine industry in one period of park-A (park $A)$ and two periods of park $B$. All the short chain PFASs $(C<8)$ and chlorinated polyfluorinated ether sulfonate acid (F-53B) in the park $A$ and $B$ were detected with $100 \%$. The first three detected substances of perfluorobutane sulfonate (PFBS), perfluorobutanoic acid (PFBA) and perfluorooctanoic acid (PFOA) were the predominant substances. The contamination status in wet season (WS) is stronger than in dry season (DS) in park B. Total concentration of PFASs( $\sum$ PFASs) in park A presented the increasing tendency following the groundwater flow direction whereas this rule was limited to reflect in all periods of park B. Applying different relative source contribution (RSC) of $100 \%$ and $20 \%$ to assess this risk of different aged group bodies from PFASs, the result was that all PFASs $(4 \leq \mathrm{C} \leq 7$ or $9 \leq \mathrm{C} \leq 12)$ while perfluorooctane sulfonate (PFOS) and PFOA $(C=8)$ were identified to the low risk quotient (RQ) contribution. Mixed $R_{\text {mix }}$ value mainly relies on the PFOA and PFOS with a larger contribution rate of $80-$ $90 \%$. All assessed cases (Case 1, Case2, Case3 and Case4) to all aged groups reveal the infants were to be vulnerable to PFASs influence, followed by the children, teenagers and adults, respectively.

\section{Introduction}

Per- and polyfluoroalkyl substances (PFASs) are a class of artificial compounds with a unique stability, surface activity, thermal stability, amphiphilic nature and have been used in various aspects of industry and household products, including surfactants, surface protectors, pesticides, lubricants, and performance chemicals(Gomez-Canela et al. 2012, Lee et al. 2020, Ssebugere et al. 2020, Xu et al. 2021). Synchronously with the industrial production and human implication in daily life, this persistent organic pollutant of PFASs have been detected in multiple environmental media, such as air(Zhao et al. 2020), soil(Shigei et al. 2020, Zhang et al. 2020), water body(Gagliano et al. 2020, Yang et al. 2011, Zheng et al. 2020), plants(Zhang et al. 2020), animals(Ahrens et al. 2016, Ahrens et al. 2010, Zhao et al. 2011) and even human blood serum(Xie et al. 2021). PFASs were divided into short and long chain according to the different chain length ( $\mathrm{C}<8$ or $\mathrm{C} \geq 8$ ), then perfluoroalkyl sulfonates (PFSAs) and perfluoroalkyl carboxylates (PFCAs) were classified by different functional groups of $-\mathrm{SO}_{3} \mathrm{H}$ and $-\mathrm{COOH}$ (Ahrens et al. 2010, Awad et al. 2011, Lindstrom et al. 2011). Although PFASs and its derivatives have many good properties and stabilities, an increasingly crucial concern of ecological influence and health issue has been implemented in the early 2000s, mainly based on two common individual-PFASs both perfluorooctanoic acid (PFOA) and perfluorooctane sulfonate (PFOS). Noticeably, PFOA, its salts and the related compounds were officially added in Annex B of POPs in 2019 (UNEP, 2019). As regulatory restrictions of long-chain PFASs were established, new alternatives of short-chain individual-PFASs and related derivatives were continually detected. These substances with long term accumulation in environment will incur body organs to suffer a vary of diseases e.g. respiratory tract, kidney, skin, reproductive and even potentially cancer-causing(Fu et al. 2014, Li et al. 2020b, Xie et al. 2021). In addition, according to the related studies in recent years, the concentration of PFASs in environmental medium were hard to degrade by microbes or bacteria, and bio- degrade by plants. The consequence may cause that PFASs will exist in environmental system and then enter into food web, seriously affect the human health.

The ground and surface water are the most precious resource due to the fact that human usually regard it as drinking source or make use of it to irrigate vegetables and fruits. But in recent years, a large scale of aqueous environment is heavily affected by PFASs, and its concentration in waterbody environment poses the risk to human health(Gobelius et al. 2018). Different PFASs sources were frequently identified in which industrial production of fluorine-containing have the largest distribution e.g. fluorine chemical industry, fluorine products manufacturing, fire protection, etc. Then related to previous studies, Gobelius et al (2018) found PFASs concentrations from 41 groundwater samples were detected with the highest $\sum$ PFASs concentration $\left(6,400 \mathrm{ng} \mathrm{L}^{-1}\right)$, associated with these areas of firefighting training sites including air fields and military areas. Meanwhile, irrigation water samples of 21 PFASs in 2019 were detected that the maximum concentrations of $369.9 \mathrm{ng} \cdot \mathrm{L}^{-1}$ around fluorine chemical park in Jiangsu province, China(Gagliano et al. 2020). Traditional wastewater equipment is difficult to completely removal the PFASs and consequently, released into receiving environment, which is a concern for the possible presence of these compounds in water used for the potable supply production(Gagliano et al. 2020, Xie et al. 2021). PFASs in water body will be ingested resulting from its abundant stability and environmental persistence. Especially, short-chain compounds enormously strengthen many learner's attention, due to their use as an alternative to long-chain ones, and their high mobility in aquatic environment(Gobelius et al. 2018, Pan et al. 2019, Qi et al. 2016).

Two parks in Fuxin (Liaoning province, China) are famous deriving from rich mineral conditions and resources (calcium fluoride, $\mathrm{CaF}_{2}$ ) where one park has been abandoned and another one is on service now. The intensive industries have generated local PFASs

Page 2/15 
contamination in relatively serve contamination degree, so the number of researches have also been implemented to focus on this area(Bao et al. 2020, Chen et al. 2018). But the surface and the ground water are only limited to study pollution status and concentration analysis without assessing water body risk to local residents, especially in groundwater as drinking source. Local residents frequently regard polluted water as drinking source though the industrial area was away from residential area, but water flow will accelerate the migration of PFASs. Thus, the health risk assessment basing on different aged groups is indispensable to evaluate the influence of PFASs in ground water to local residents. Importantly, individual and mixed risk of PFASs including short and long chain can provide the accurate value to measure the risk degree and single out priority pollutants for prevention and control.

Thus, the objective of our study is to (1) identify PFASs concentration and profile in the surface and ground water. (2) definite PFASs spatial distribution around regional area of two parks. (3) assess the individual and mixed of risk from short and long chain PFASs in ground water to the local residents. Through our study, it is expected to provide theoretical basis and scientific guidance of PFASs pollution for local policies departments or makers.

\section{Materials And Methods}

\subsection{Chemicals and regants}

All the chemicals and standards were of chromatographically pure grade. The target PFAS analytes were purchased from Absolute Standards (Hamden, CT, USA), including polyfluorinated ether sulfonate acid (F-53B), 13 PFCAs of perfluorobutanoic acid (PFBA), perfluoropentanoic acid (PFPeA), perfluorohexanoic acid (PFHxA), perfluoroheptanoic acid (PFHpA), PFOA, perfluorononanoic acid (PFNA), perfluorodecanoic acid (PFDA), perfluoroundecanoic acid (PFUdA), perfluorododecanoic acid (PFDoA), perfluorotridecanoic acid (PFTrDA), perfluorotetradecanoic acid (PFTeDA), perfluorohexadecanoic acid (PFHxDA) and perfluorooctadecanoic acid (PFODA), 4 PFSAs of perfluorobutane sulfonate (PFBS), perfluorohexane sulfonate (PFHxS), PFOS and perfluorodecane sulfonate (PFDS). Standard information and the selection of target compounds are given in the supporting information (SI).

\subsection{Sample collection and analysis}

All water sampling works were carried out in September (wet season, WS) and November (dry season, DS) of 2019 in the two parks of Fuxin, China. However, a number of times (WS) in park A was only collected due to the relocation and departure of local residents. Thirty-three samples were collected in park A and park B of DS and WS. The surface water samples mainly come from local river (Xi river tributaries) and pond, and the ground water samples were collected from resident's well. The ground water samples at a depth of 7-10 $\mathrm{m}$ and surface samples at a depth of $10-20 \mathrm{~cm}$ were collected by using $1.5 \mathrm{~L}$ methanol-rinsed polyethylene (PE) bottles with polypropylene (PP) screw caps. All water samples were stored by portable refrigerator $\left(\sim 5^{\circ} \mathrm{C}\right)$ and avoided light. Water sample pretreatment methods mainly come from Chen et al (2018) and Bao et al (2019). Oasis WAX cartridges (500 mg, $6 \mathrm{~mL}$, Waters, USA) were applied to extract PFASs and extracts of the groundwater and the surface water samples were analyzed via high performance liquid chromatography triple quadrupole mass spectrometer (HPLC-MS/MS). The WAX SPE cartridges were conditioned with $5 \mathrm{~mL}$ methanol containing $0.1 \%$ ammonium hydroxide, $5 \mathrm{~mL}$ methanol and $5 \mathrm{~mL}$ Milli-Q water. Then, the water samples were loaded onto the cartridges at a rate of $1 \mathrm{drop} / \mathrm{s}$. The cartridges were washed with $5 \mathrm{~mL}$ of $2.5 \mathrm{mM}$ acetic acid/ ammonium acetate $(\mathrm{pH}=4)$, and then centrifuged for $10 \mathrm{~min}$ at $3000 \mathrm{rpm}$ to remove residual water. The ionizable compounds were eluted with $5 \mathrm{~mL}$ methanol and $5 \mathrm{~mL}$ methanol containing $0.1 \%$ ammonium hydroxide. The eluent was concentrated under nitrogen and dissolved with $1 \mathrm{ml}$ acetonitrile for instrumental analysis (Bao et al. 2020, Cao et al. 2019, Chen et al. 2018).

\subsection{QA/QC}

The field blank to eliminate the concentration influencing from sampling bottle and series of laboratory Milli-Q water were added into the sampling bottle in order to determine this free blank. Procedural blanks were identified to every batch of samples and it will not be detected (nd) or lower than the limits of quantification (LOQs) in order to ensure data accuracy and method availability. LOQ were defined as the minimum injection volume of reproducible measurement peak area within $\pm 20 \%$ of repeated injection. The LOD and LOQ for each PFASs were given in SI. The PFASs concentrations were identified to a concentration series of $0,0.01,0.1,1,5,10,50,100$, and $500 \mu \mathrm{g} \cdot \mathrm{L}^{-1}$ to draw the standard curve $\left(\mathrm{R}^{2}>0.99\right)$. In addition, the IS was added to each sample to assess accuracy by the recovery of the analytes, and recoveries acceptable range needs to be less than $20 \%$.

\subsection{Statistical analysis}


To further figure out this health effect of PFASs to the local resident is so important that an original quotient method was applied in our study. The new risk quotient (RQ) methodology coming from Thomaidi et al study (2020), was applied to assess human health risk from the groundwater. This method mainly was different from the other RQ assessment method by using the relative source contribution factor (RSC, the percentage of the exposure due to the drinking water consumption, and the value for $100 \%$ and $20 \%$, $\mathrm{RSC}_{100 \%}$ and $\mathrm{RSC}_{20 \%}$ ). The RQ calculation of individual PFASs was given for equation (1)- (3):

$$
\mathrm{DWEL}_{100 \%}=\frac{\mathrm{ADI} \times \mathrm{BW}}{\mathrm{DWI} \times \mathrm{AB} \times \mathrm{FOE}}
$$

$$
\mathrm{DWEL}_{20 \%}=\frac{0.2 \times \mathrm{ADI} \times \mathrm{BW}}{\mathrm{DWI} \times \mathrm{AB} \times \mathrm{FOE}}
$$

$$
\mathrm{RQ}_{100 \%} / \mathrm{RQ}_{20 \%}=\frac{\mathrm{C}}{\mathrm{DWEL}_{100 \%} / \mathrm{DWEL}_{20 \%}}
$$

In our study, similarity RSC of $100 \%$ and $20 \%$ will be taken into consideration for different aged groups of infants (birth- 3 months, $3-6$ months, 6-12 months, 1-2years, 2-3years), children (3-6years, 6-11years), teenagers (11-16years, 16-21years) and adults ( $>21$ years), respectively. Meanwhile, the max and mean concentration of PFASs were calculated with RSC of $100 \%$ as case 1 and case 2 , then with RSC of $20 \%$ as case 3 and case 4 (Table S4) in order to identify the risk from different aged groups. On the other hand, multiple PFASs in the practical water body should be assessed by mixture risk of PFASs which mainly was used to evaluate the risk value of various PFASs in groundwater. $R Q$ value of mixed PFASs $\left(R Q_{\text {mix }}\right)$ was calculated by the sum of single individual-PFASs $\left(R Q_{\text {mix }}\right)$ and listed in Equation (4):

$$
\mathrm{RQ}_{\text {mix }}=\sum_{\mathrm{i}=1}^{\mathrm{n}} \mathrm{RQ}_{\mathrm{i}}=\sum_{\mathrm{i}=1}^{\mathrm{n}} \frac{\mathrm{C}_{\mathrm{i}}}{\mathrm{DWEL}_{\mathrm{i}}}
$$

Where

$\mathrm{ADI}\left(\mu \mathrm{g} / \mathrm{kg} \mathrm{day}^{-1}\right)$ is the acceptable daily intake without significant health risk, $\mathrm{BW}(\mathrm{kg})$ represents percentile body weight, DWI $\left(\mathrm{L}\right.$ day $\left.{ }^{-1}\right)$ is drinking water intake in daily life, $A B$ defines the gastrointestinal absorption rate (assumed to be equal to 1), FOE is the frequency of exposure ( 350 days $/ 365$ days $=0.96$ ) and $n$ in Equation $(4)$ is to assess the potential risk for human health related to mixed PFASs contamination. These parameter values can be found in Table S5. The evaluation standard of RQ methodology is by the comparation between $R Q$ value and $0.2 \& 1$. If $R Q$ is higher than $0.2(R Q=1)$ which indicates local water body has an adverse effect (extremely serious effect) on human health and if the RQ is lower than 0.2, it indicates that the harmful influence can be neglected(Cao et al. 2019, Etchepare \&van der Hoek 2015, Sun et al. 2018, Thomaidi et al. 2020).

\subsubsection{Data analysis}

Statistical analysis and significance test were analyzed by IBM SPSS statistics 22. Origin 2017 and Arcgis 10.7 are to make table and figure in our study.

\section{Results And Discussion}

3.1 Concentration and profiles 
Eighteen individual PFASs all were analyzed in park A and B (Fig. 2). All the short chain PFASs $(C<8)$ and F-53B in park A and B all were detected with the rate of $100 \%$. Generally, the contamination status of PFASs in park A was higher than park B. Due to the industrial production of two parks, the total concentration of PFASs ( $\sum$ PFASs) and the main individual-PFASs concentration exceeds other regions of Taihu lake(Ma et al. 2018), West lake(Lu et al. 2018), Jiaozhou bay (Han et al. 2020)and Bohai sea(Chen et al. 2017) (Table S6). In addition, comparing with other drinking water sources of central eastern China, mainly detected substances (PFBA, PFHxA and PFOA) and $\sum$ PFASs were lower than our studies area(Lu et al. 2018). Some previous studies revealed serious contamination degree in local area. The range of $\sum$ PFASs(15 target substances) in 2018 was $87-18,000 \mathrm{ng} \cdot \mathrm{L}^{-1}$, and PFBA was $16-12,000 \mathrm{ng} \cdot \mathrm{L}^{-1}$, followed by PFBS (4-5,800 $\left.\mathrm{ng} \cdot \mathrm{L}^{-1}\right)$ and PFOA with (16-1,400 $\left.\mathrm{ng} \cdot \mathrm{L}^{-1}\right)$, respectively(Chen et al. 2018). Then, Bao et al (2020) detected groundwater as local irrigation source that $\sum$ PFASs $\left(10\right.$ target substances) has exceed $35 \mathrm{\mu g} \mathrm{L}^{-1}$, the mainly detected PFASs concentrations of PFBS $\left(32.40 \mu \mathrm{g} \mathrm{L}^{-1}\right)$ and PFOA $\left(2.47 \mu \mathrm{g} \mathrm{L}^{-1}\right)$ (Bao et al. 2020)(Table S2).

In park A, $\sum$ PFASs varies from $72.49 \mathrm{ng} \cdot \mathrm{L}^{-1}$ in Ga7 to $68,142.16 \mathrm{ng} \cdot \mathrm{L}^{-1}$ in Ga10 and the mean concentration degree was $9,104.03 \mathrm{ng} \cdot \mathrm{L}^{-1}$. Although park $A$ has been forbade to carry out industrial production and lacks out obvious point pollution for a long time, PFASs were detected with the high concentration due to itself environmental accumulation and stability of persistent organic pollutant (POPs)(Naile et al. 2013, Nascimento et al. 2018, Ojemaye \&Petrik 2019). Short chain PFASs concentration was obviously stronger than long chain PFASs. The highest mean concentration of PFBS was detected with $8,734.27 \mathrm{ng} \cdot \mathrm{L}^{-1}$, followed by PFOA $\left(1,563.13 \mathrm{ng} \cdot \mathrm{L}^{-1}\right), \mathrm{PFBA}(400.41$ $\left.\mathrm{ng} \cdot \mathrm{L}^{-1}\right), \mathrm{PFHxA}\left(136.09 \mathrm{ng} \cdot \mathrm{L}^{-1}\right)$ and PFHxS $\left(106.87 \mathrm{ng} \cdot \mathrm{L}^{-1}\right)$, respectively (Table 1). The larger concentration contribution of C4-C7 (16\%-63\%) and C8 (34\%-83\%) were stronger than C>8 PFASs ( $\leq 3 \%$ ) (Fig 3a). Raining activity in surface and ground system play an important impact on the migration of PFASs(Ahrens et al. 2016, Xu et al. 2021). The ground water flow and surface water supply will cause the increasing of PFASs concentration in superficial aquifer while along with time variation, a mass of storm runoff will dilute high PFASs concentration( $\mathrm{Li}$ et al. 2020a). PFSAs with functional groups of $-\mathrm{SO}_{3} \mathrm{H}$ in surface and groundwater emerge a larger concentration ratio (51\%-80\%) more than PFCAs with - $\mathrm{COOH}(20 \%-49 \%)$. In especial, PFSAs in Ga2 with $80 \%$ concentration contribution whereas PFCAs only accounts for $20 \%$ (Fig. S1a).

In park B, the PFASs contamination status in groundwater of WS is far stronger than in DS. The mean concentration was calculated with 801.68 and $714.64 \mathrm{ng} \cdot \mathrm{L}^{-1}$ in WS and DS, respectively. The mean concentration of surface water was analyzed with $136.39 \mathrm{ng} \cdot \mathrm{L}^{-1}$ in WS whereas mean concentration has reached $1,257.60 \mathrm{ng} \cdot \mathrm{L}^{-1}$ in DS. Similarly, PFBS, PFBA and PFOA were also the mainly detected substances in surface and ground water of WS or DS (Table 1). The serve contamination of short chain PFASs in recent years results from their vast using as long chain alternatives. As Bao et al study indicated that PFBA, PFPeA and PFHxA were used to be the alternatives of PFOA and PFBS is the main substitute of PFOS(Bao et al. 2020). This sampling site of Rbb1 locates on the agricultural area, detected with serious individual concentration and $\sum$ PFASs due to long term reception place from agricultural activities, even pesticides and fertilizers using (Fig.1, Fig. $2 \mathrm{c}$ and Table S7). Surface water will accelerate the migration of pollutants, and especially the supply also carries PFASs into groundwater(Chiavola et al. 2020, Feng et al. 2020). The PFOS substitute of F-53B was detected with low contamination degree, whereas concentration in surface water was higher in the groundwater. What in mentioned that long chain PFASs ( $(\geq 10)$ that PFDS, PFUdA, PFDoA, PFTeDA, PFTrDA and PFODA were detected certain concentration in all water body of DS without appearing in WS (nd). C4-C7 and C8 of PFASs show higher concentration contribution than C9 of PFASs in the surface and ground water of WS and DS. There was a difference that concentration rate of C4-C7 PFASs in DS (range of 64\%-98\%) was far lower than in WS (range of 95\%-98\%) (Fig. 3b,c). Time difference was better reflected on short chain PFASs than long chain PFASs. The reason was that short chain PFASs with greater hydrophobicity and lower lipophilicity will tend to be affected by season variation while long chain PFASs were adsorbed by organic matter and the suspended material in sediment(Ahrens et al. 2016, Gagliano et al. 2020, Xu et al. 2021, Zheng et al. 2020). For function groups, the concentration contribution of PFSAs was far than PFCAs in park B (Fig. S1b). Chemical groups between $-\mathrm{SO}_{3} \mathrm{H}$ and $-\mathrm{COOH}$ make PFASs in water possess the environmental behavior abundantly(Gobelius et al. 2018).

In our study, PFASs concentration have closely negative relationship with chain length in park A and B (Fig. S2). PFASs with unique physicochemical structure tend to dissolve in water body and migrate with a long distance. In addition, the functional groups are also an important factor(Liu et al. 2019). Functional groups difference between PFSAs and PFCAs are limited to affect by the water load variation in different term (Fig S1). Although other non-point sources may contribute to PFASs in surface and groundwater, the park production is the major contamination source. In addition, industrial emission in different time also affects PFASs profiles by discharge of sewage because traditional equipment is hard to removal effectively.

3.2 Spatial distribution

Page 5/15 
The source of PFASs in the groundwater may be assumed to two pathways. One was that industrial waste water was directly discharged into groundwater system and another was the ground pollutant source by some environmental behavior of the surface runoff, surface water recharge and rainwater deposition, which will carry PFASs and its precursors into groundwater(Ahrens et al. 2010, Bao et al. 2020, Blaine et al. 2014, Cao et al. 2019, Chen et al. 2015, Miranda et al. 2021). The spatial distribution of the pollutants is not only related to its chemical properties and structures but also the environmental factor and geographical condition should be taken into consideration(Catherine et al. 2019, Chen et al. 2015, Choi et al. 2011, Gao et al. 2020). Especially in intensive industry region, river direction, groundwater flow direction and agricultural activities, all closely affect the distribution of PFASs. In park A, mainly detected substances of individual PFASs and $\sum$ PFASs show an increasing tendency along with the groundwater flow direction (Fig. S3). This result indicates that the groundwater flow direction is the key element to the PFASs polluted distribution. In addition, the mainly detected individual-PFASs of PFBA, PFBS, PFHpA, PFHxA, PFHxS, PFPeA and PFOA is the same with the tendency of $\sum$ PFASs (Fig. S3). Different industrial types are the critical reason of causing PFASs distribution but PFASs in water body was also influenced by the upstream waterbody(Lee et al. 2020). Although industrial process is the main source, the groundwater flow direction also possesses a significant function to PFASs distribution and migration in park A. Other studies evidenced that the PFASs not only comes from the point source but also attributes to water mass migration(Lee et al. 2020).

In park B, this distribution of PFASs concentration was limited to be reflected, and the dominating detected substances and $\sum$ PFASs also lack out obviously increasing or decreasing concentration tendency along with the groundwater flow direction. The concentration of PFASs in the downstream water is slightly higher than the upstream along with the groundwater flow direction but this tendency is largely weak (Fig S4). Some sites show abnormal concentration due to the location distribution of the waste water treatment plant (WWTP) and fire station (FS). These places all influence the spatial distribution of PFASs. Current biotreatment in WWTP can remove parts of PFNA, PFHXA, and PFPeA but has limited ability to treat other PFASs(Knutsen et al. 2019, Xu et al. 2021). In addition, FS as important pollution point has been widely reported. Graetz et al. stated that the past firefighting water additives were found to contain the PFASs that could persist in the environment resulting in the potential adverse effects to the biota(Graetz et al. 2020). Importantly, a FS in the park B is the most polluted sources. Daily training and drills will widely release the PFASs precursors into the environment (Gobelius et al. 2017, Graetz et al. 2020). Different areas were polluted by the point and the non-point source of PFASs and finally these substances entering into the groundwater system, even migrate to other places(Li et al. 2020a, Li et al. 2020b). PFASs distribution was hard to reveal the increasing and decreasing tendency due to tremendously industrial emission with different states of the waste air and water in park B. The industrial process and the production possess a strong effect on the distribution of PFASs far than geographical factor.

\subsection{Health risk assessment}

Environmental pollutants will enter into and finally accumulate in the human body by direct drinking(Gobelius et al. 2018). In our study, the groundwater as main drinking source is a serve exposure to local residents. The short chain PFASs were mainly detected in two parks whereas part of PFASs lacks out related evaluation messages, still all individual PFASs is hard to assess the health risk. Hence, combining with the mainly detected substances and available toxicity data, PFBA(C4), PFHpA(C7), PFHxA(C6), PFHxS(C6), PFOA(C8), PFOS(C8), PFNA(C9), PFUdA(C11) and PFDoA(C12) were assessed, respectively.

\subsubsection{Individual PFASs (C $\leq 8)$}

Along with the increasing of the aged groups, the RQ value show a decreasing tendency in park A and park B-WS and DS. This reveals that PFASs risk have a more remarkable influence on the infants and the children than the teenagers and the adults. $\mathrm{RQ}_{100 \% / 20 \%} \mathrm{value}$ has a positive relationship with PFASs concentration in the water body and the negative relationship with DWEL. In park A, the highest DWEL value appears in PFBS $\left(17,820,547.09-3,270,348.84 \mathrm{~L} \cdot\right.$ day $\left.^{-1}\right)$, followed by PFHpA and PFHxA $\left(654,069.01-3,564,109.42 \mathrm{~L} \cdot\right.$ day $^{-}$ $\left.{ }^{1}\right)$, respectively. But these three substances lack out determining the highest health risk whereas PFOA possesses a largest RQ value, followed by PFOS (Table S8). $\mathrm{RQ}_{100 \% / 20 \%}$ value of all assessed substances in park $A$ is higher than park $B$ and especially part of the $\mathrm{RQ}_{100 \% / 20 \%}$ value in park $A$ has exceeded 1 (Table S8). The $\mathrm{RQ}_{100 \% / 20 \%}$ value of PFBA, PFHpA, PFHxA and PFHxS in case 1-4 were in the reasonable range and this demonstrates that the max and the mean concentration of four substances is limited to exceed the RQ reference line of 0.2 and 1 . Short chain PFASs $(C \leq 7)$ in groundwater is hard to constitute the health risk to local residents. But PFOA and PFOS in part of sampling sites exceeding $R Q=0.2$, even $R Q=1$, has given rise to harm human body in case $1-4$. The $R Q_{100 \% / 20 \%}$ value of PFOA in case 1 and case 3 were in the range of 0.26-1.44 and 1.32-7.20 for aged groups, and the range of 0.23-0.64 for birth 6years in case 4 (Fig. 4a). What counts is that total PFASs contamination degree is hard to pose health risk in case 2 for all aged groups. Causally, the water flow is the main impact to take away PFASs for the part of sampling sites with high concentration and this 
will dilute PFASs concentration, even decrease the toxic effect(Hongkachok et al. 2018, lkkere et al. 2018, Jin et al. 2015). The ADI value difference between PFOS $\left(0.15 \mu \mathrm{g} / \mathrm{kg} \mathrm{day}^{-1}\right)$ and PFOA $\left(1.5 \mu \mathrm{g} / \mathrm{kg}\right.$ day $\left.{ }^{-1}\right)$ has exceeded 1 orders of magnitude. However, the concentration of PFOS is 1-2 magnitude lower than PFOA which causes the $R Q_{100 \% / 20 \%}$ value of PFOS is hard to exceed PFOA. The $R_{100 \% / 20 \%}$ value of PFOS in case 1-2 and 4 was difficult to exceed $R Q=0.2$ expect for case 3 , which is similar to PFOA. For different periods in park $B$, the degree of $R Q_{100 \% / 20 \%}$ value in WS is higher in DS due to the high concentration was detected in WS (Fig $4 b, c$ and Table S8). Based on all terms of different aged groups except the case 3 , only PFOA in WS exceeds the control line of RQ $=0.2$ but is not exceed $R Q=1$. The range of $R_{20}$ value for PFOA in case 3 was $0.22-0.62$ that the max value of 0.62 for birth-3 months and the mix value of 0.22 for 3-6 years (Fig 4 b,c). Many studies have revealed that PFASs can harm human body but different types of PFASs have various effect mechanisms(Graetz et al. 2020, He et al. 2018, Li et al. 2019, Miranda et al. 2021). Local residents regard the polluted water as drinking source, having larger exposure opportunity of touching these toxic substances.

\subsubsection{Individual PFASs (C>8)}

Long chain PFASs $(C>8)$ were detected with lower concentration than short chain PFASs, which means the low concentration was also related to lower RQ value. Three individual-PFASs of PFNA(C9), PFUdA (C11) and PFDoA(C12) were assessed. The result reveal that three long chain PFASs is hard to pose potential risk to the local residents. The range of RQ value is that the negligible (ng)- 0.002 , ng0.0001 and ng- 0.006 for PFNA, PFUdA and PFDoA, respectively (Table S8). These max $R Q_{100 \% / 20 \%}$ value from three long chain PFASs were lower than 0.2 while the magnitude difference ranges from 1 to 3 . Then in our study, extreme conditions are considered to perform operations to initially assess the risk to the human health. The group of birth-3 months can be easily affected by short chain PFASs in environmental media. If three assessed substances will cause health risk basing on the line of $R Q=0.2$, the concentration of $5.37,6.53$ and $0.13 \mathrm{ng} \cdot \mathrm{L}^{-1}$ for PFNA, PFUdA and PFDoA were calculated, respectively. There was a huge difference of 1-2 magnitudes between the predicted and actual concentration. Thus, the long chain PFASs for assessed substances were difficult to threaten the human body.

\subsubsection{Mixed risk of PFASs}

The mixed risk assessment was increasingly concerned due to the different individual PFASs existing in the environmental media. But the related assessing method is limited to develop resulting from the complex mechanism in current environment system. The most serious situation appears in the park $A$ of $R_{\text {mix }}(4 \leq C \leq 12)=8.07$ for birth-3 months (Table S6). $\sum P F A S s(4 \leq C \leq 7)$ and $\sum$ PFASs $(9 \leq C \leq 12)$ were hard to reach the line of $R Q=0.2$, and $R Q_{\text {mix }}$ of these substances were limited to exceed that $R Q=1$ (Table $S 7$ ). Expect for PFOS and PFOA, all PFASs ( $4 \leq \mathrm{C} \leq 7$ or $9 \leq \mathrm{C} \leq 12)$ were analyzed with lower RQ contribution. Especially, the risk of the individual PFASs with low $R Q$ value without exceeding $R Q=0.2$, then the $R Q_{\text {mix }}$ value was hard to possess the risk to all aged groups. In this process, PFOS and PFOA risk values determine the crucial factor to the size of $R_{\mathrm{mix}}$. In other words, if the risk from PFOS and PFOA can be effectively impaired, the $R Q_{\text {mix }}$ of all the individual-PFASs will deeply decrease. Mixed $R_{Q_{m i x}}$ value mainly relies on the PFOA and PFOS contribution rate (80\%-90\%). This indicates that PFOA and PFOS should be taken into consideration and listed as priority control. Notably, $\mathrm{RQ}_{\mathrm{mix}}$ value from the group of teenagers (16-21 years) appears a small spike relative to two adjacent groups both 11-16 years and $>21$ years. In comparison with the infant and child, teenager group is the third group which is influenced by PFASs due to the weight and drinking intake.

\section{Conclusion}

Eighteen individual PFASs were analyzed in surface water and groundwater, then the concentration profiles, spatial distribution and the health risk assessment for different aged groups was studied in two different parks. PFBS, PFBA and PFOA were the predominant substances in surface and ground water, meanwhile, $\sum$ PFASs in park A was higher than in park B. Water loads in different periods have a crucial impact on PFASs concentration and profiles variation. PFSAs and PFCAs also appear negative concentration trend in WS or DS. In addition, $\sum$ PFASs and part of high concentration substances(PFBA, PFBS, PFHpA, PFHxA, PFHxS, PFOA and PFPeA) appear the increasing tendency along with the groundwater flow direction in park A. However, due to the influence of industrial process and emission, the concentration trend was limited to reflect in park B. Health risk assessment result was that short and long chain PFASs except for the PFOS and PFOA were hard to possess the serious risk to the local resident based on the line of $R Q=0.2$. The serious health risk from PFOS and PFOA were found with a larger contribution to the mixed $R_{Q_{m i x}}$ value. All assessed cases to all aged groups reveal the infants were vulnerable to the PFASs influence, followed by the children, teenagers and adults, respectively.

\section{Declarations}




\section{Ethics approval and consent to participate}

Not applicable.

\section{Consent for publication}

Not applicable.

\section{Availability of data and materials}

All data generated or analysed during this study are included in this published article and its supplementary information files.

\section{Competing interests}

The authors declare that they have no competing interests

\section{Funding}

This work was supported by the Basic research project of science and technology of Liaoning Provincial Department of Education (LJ2019JL029) and Integration of ecosystem restoration and management technologies and demonstration of ecological industry development in northwest Liaoning Province (2020JH1/10300006).

\section{Author contributions}

Authors contributed to this work as follows: J. X. Tang (conceptualization, formal analyses, funding acquisition, methodology, Investigation, supervision, writing (original, review and editing) ), Y. L. Zhu (conceptualization, data curation, analyses, methodology, writingoriginal draft), Y. Li (conducted the experiment, data curation, investigation, methodology, supervision, writing-original draft), B. Xiang (conducted the experiment, data curation, writing-review and editing), T. Ting (data curation, analyses, methodology, writingoriginal draft), L. Y. Lv (conceptualization, funding acquisition, writing-review and editing), Q. Luo (conceptualization, methodology, supervision, writing-review and editing). All authors read and approved the final manuscript.

\section{References}

1. Ahrens L, Taniyasu S, Yeung LW, Yamashita N, Lam PK, Ebinghaus R (2010) Distribution of polyfluoroalkyl compounds in water, suspended particulate matter and sediment from Tokyo Bay. Japan Chemosphere 79:266-266 720

2. Ahrens L, Gashaw H, Sjoholm M, Gebrehiwot SG, Getahun A, Derbe E, Bishop K, Akerblom S (2016) Poly- and perfluoroalkylated substances (PFASs) in water, sediment and fish muscle tissue from Lake Tana, Ethiopia and implications for human exposure. Chemosphere 165:352-357

3. Awad E, Zhang X, Bhavsar SP, Petro S, Crozier PW, Reiner EJ, Fletcher R, Tittlemier SA, Braekevelt E (2011) Long-term environmental fate of perfluorinated compounds after accidental release at Toronto airport. Environ Sci Technol 45:8081-8089

4. Bao J, Li CL, Liu Y, Wang X, Yu WJ, Liu ZQ, Shao LX, Jin YH (2020) Bioaccumulation of perfluoroalkyl substances in greenhouse vegetables with long-term groundwater irrigation near fluorochemical plants in Fuxin, China. Environ Res 188:109751

5. Blaine AC, Rich CD, Sedlacko EM, Hyland KC, Stushnoff C, Dickenson ER, Higgins CP (2014) Perfluoroalkyl acid uptake in lettuce (Lactuca sativa) and strawberry (Fragaria ananassa) irrigated with reclaimed water. Environ Sci Technol 48:14361-14368

6. Cao X, Wang C, Lu Y, Zhang M, Khan K, Song S, Wang P, Wang C (2019) Occurrence, sources and health risk of polyfluoroalkyl substances (PFASs) in soil, water and sediment from a drinking water source area. Ecotoxicol Environ Saf 174:208-217

7. Catherine M, Nadege B, Charles P, Yann A (2019) Perfluoroalkyl substances (PFASs) in the marine environment: Spatial distribution and temporal profile shifts in shellfish from French coasts. Chemosphere 228:640-648

8. Chen H, Zhang C, Han J, Sun R, Kong X, Wang X, He X (2015) Levels and spatial distribution of perfluoroalkyl substances in China Liaodong Bay basin with concentrated fluorine industry parks. Mar Pollut Bull 101:965-971

9. Chen H, Wang X, Zhang C, Sun R, Han J, Han G, Yang W, He X (2017) Occurrence and inputs of perfluoroalkyl substances (PFASs) from rivers and drain outlets to the Bohai Sea, China. Environ Pollut 221:234-243

10. Chen H, Yao Y, Zhao Z, Wang Y, Wang Q, Ren C, Wang B, Sun H, Alder AC, Kannan K (2018) Multimedia Distribution and Transfer of Per- and Polyfluoroalkyl Substances (PFASs) Surrounding Two Fluorochemical Manufacturing Facilities in Fuxin, China. Environ 
Sci Technol 52:8263-8271

11. Chiavola A, Di Marcantonio C, Boni MR, Biagioli S, Frugis A, Cecchini G (2020) Experimental investigation on the perfluorooctanoic and perfluorooctane sulfonic acids fate and behaviour in the activated sludge reactor. Process Saf Environ Prot 134:406-415

12. Choi HG, Moon HB, Choi M, Yu J (2011) Monitoring of organic contaminants in sediments from the Korean coast: Spatial distribution and temporal trends (2001-2007). Mar Pollut Bull 62:1352-1361

13. Etchepare R, van der Hoek JP (2015) Health risk assessment of organic micropollutants in greywater for potable reuse. Water Res 72:186-198

14. Feng X, Ye M, Li Y, Zhou J, Sun B, Zhu Y, Zhu L (2020) Potential sources and sediment-pore water partitioning behaviors of emerging per/polyfluoroalkyl substances in the South Yellow Sea. J Hazard Mater 389:122124

15. Fu Y, Wang T, Wang P, Fu Q, Lu Y (2014) Effects of age, gender and region on serum concentrations of perfluorinated compounds in general population of Henan. China Chemosphere 110:104-110

16. Gagliano E, Sgroi M, Falciglia PP, Vagliasindi FGA, Roccaro P (2020) Removal of poly-and perfluoroalkyl substances (PFAS) from water by adsorption: Role of PFAS chain length, effect of organic matter and challenges in adsorbent regeneration. Water Res $171: 115381$

17. Gao L, Liu J, Bao K, Chen N, Meng B (2020) Multicompartment occurrence and partitioning of alternative and legacy per- and polyfluoroalkyl substances in an impacted river in China. Science of The Total Environment 729

18. Gobelius L, Lewis J, Ahrens L (2017) Plant Uptake of Per- and Polyfluoroalkyl Substances at a Contaminated Fire Training Facility to Evaluate the Phytoremediation Potential of Various Plant Species. Environ Sci Technol 51:12602-12610

19. Gobelius L, Hedlund J, Durig W, Troger R, Lilja K, Wiberg K, Ahrens L (2018) Per- and Polyfluoroalkyl Substances in Swedish Groundwater and Surface Water: Implications for Environmental Quality Standards and Drinking Water Guidelines. Environ Sci Technol 52:4340-4349

20. Gomez-Canela C, Barth JA, Lacorte S (2012) Occurrence and fate of perfluorinated compounds in sewage sludge from Spain and Germany. Environ Sci Pollut Res Int 19:4109-4119

21. Graetz S, Ji M, Hunter S, Sibley PK, Prosser RS (2020) Deterministic risk assessment of firefighting water additives to aquatic organisms. Ecotoxicology 29:1377-1389

22. Han T, Gao L, Chen J, He X, Wang B (2020) Spatiotemporal variations, sources and health risk assessment of perfluoroalkyl substances in a temperate bay adjacent to metropolis, North China. Environmental Pollution 265

23. He X, Li A, Wang S, Chen H, Yang Z (2018) Perfluorinated substance assessment in sediments of a large-scale reservoir in Danjiangkou, China. Environ Monit Assess 190:66

24. Hongkachok C, Boontanon SK, Boontanon N, Fujii S, Tanaka S, Suzuki Y (2018) Levels of perfluorinated compounds (PFCs) in groundwater around improper municipal and industrial waste disposal sites in Thailand and health risk assessment. Water Sci Technol 2017, 457-466

25. Ikkere LE, Perkons I, Sire J, Pugajeva I, Bartkevics V (2018) Occurrence of polybrominated diphenyl ethers, perfluorinated compounds, and nonsteroidal anti-inflammatory drugs in freshwater mussels from Latvia. Chemosphere 213:507-516

26. Jin H, Zhang Y, Zhu L, Martin JW (2015) Isomer profiles of perfluoroalkyl substances in water and soil surrounding a chinese fluorochemical manufacturing park. Environ Sci Technol 49:4946-4954

27. Knutsen H, Maehlum T, Haarstad K, Slinde GA, Arp HPH (2019) Leachate emissions of short- and long-chain per-and polyfluoralkyl substances (PFASs) from various Norwegian landfills. Environ Sci Process Impacts 21:1970-1979

28. Lee YM, Lee JY, Kim MK, Yang H, Lee JE, Son Y, Kho Y, Choi K, Zoh KD (2020) Concentration and distribution of per- and polyfluoroalkyl substances (PFAS) in the Asan Lake area of South Korea. J Hazard Mater 381:120909

29. Li BB, Hu LX, Yang YY, Wang TT, Liu C, Ying GG (2020a) Contamination profiles and health risks of PFASs in groundwater of the Maozhou River basin. Environ Pollut 260:113996

30. Li P, Oyang X, Zhao Y, Tu T, Tian X, Li L, Zhao Y, Li J, Xiao Z (2019) Occurrence of perfluorinated compounds in agricultural environment, vegetables, and fruits in regions influenced by a fluorine-chemical industrial park in China. Chemosphere 225:659667

31. Li Y, Barregard L, Xu Y, Scott K, Pineda D, Lindh CH, Jakobsson K, Fletcher T (2020b) Associations between perfluoroalkyl substances and serum lipids in a Swedish adult population with contaminated drinking water. Environmental Health 19 
32. Lindstrom AB, Strynar MJ, Delinsky AD, Nakayama SF, McMillan L, Libelo EL, Neill M, Thomas L (2011) Application of WWTP Biosolids and Resulting Perfluorinated Compound Contamination of Surface and Well Water in Decatur, Alabama, USA. Environmental Science \& Technology 45, 8015-8021

33. Liu L, Liu Y, Gao B, Ji R, Li C, Wang S (2019) Removal of perfluorooctanoic acid (PFOA) and perfluorooctane sulfonate (PFOS) from water by carbonaceous nanomaterials: A review. Critical Reviews in Environmental Science Technology 50:2379-2414

34. Lu Z, Lu R, Zheng H, Yan J, Song L, Wang J, Yang H, Cai M (2018) Risk exposure assessment of per- and polyfluoroalkyl substances (PFASs) in drinking water and atmosphere in central eastern China. Environ Sci Pollut Res Int 25:9311-9320

35. Ma X, Shan G, Chen M, Zhao J, Zhu L (2018) Riverine inputs and source tracing of perfluoroalkyl substances (PFASs) in Taihu Lake, China. Science of The Total Environment 612:18-25

36. Miranda DA, Benskin JP, Awad R, Lepoint G, Leonel J, Hatje V (2021) Bioaccumulation of Per- and polyfluoroalkyl substances (PFASs) in a tropical estuarine food web. Sci Total Environ 754:142146

37. Naile JE, Khim JS, Hong S, Park J, Kwon BO, Ryu JS, Hwang JH, Jones PD, Giesy JP (2013) Distributions and bioconcentration characteristics of perfluorinated compounds in environmental samples collected from the west coast of Korea. Chemosphere 90:387-394

38. Nascimento RA, Nunoo DBO, Bizkarguenaga E, Schultes L, Zabaleta I, Benskin JP, Spanó S, Leonel J (2018) Sulfluramid use in Brazilian agriculture: A source of per- and polyfluoroalkyl substances (PFASs) to the environment. Environ Pollut 242:1436-1443

39. Ojemaye CY, Petrik L (2019) Occurrences, levels and risk assessment studies of emerging pollutants (pharmaceuticals, perfluoroalkyl and endocrine disrupting compounds) in fish samples from Kalk Bay harbour, South Africa. Environ Pollut 252:562572

40. Pan CG, Yu KF, Wang YH, Zhang W, Zhang J, Guo J (2019) Perfluoroalkyl substances in the riverine and coastal water of the Beibu Gulf, South China: Spatiotemporal distribution and source identification. Sci Total Environ 660:297-305

41. Qi Y, Huo S, Hu S, Xi B, Su J, Tang Z (2016) Identification, characterization, and human health risk assessment of perfluorinated compounds in groundwater from a suburb of Tianjin, China. Environmental Earth Sciences 75

42. Shigei M, Ahrens L, Hazaymeh A, Dalahmeh SS (2020) Per- and polyfluoroalkyl substances in water and soil in wastewaterirrigated farmland in Jordan. Sci Total Environ 716:137057

43. Ssebugere P, Sillanpaa M, Matovu H, Wang Z, Schramm KW, Omwoma S, Wanasolo W, Ngeno EC, Odongo S (2020) Environmental levels and human body burdens of per- and poly-fluoroalkyl substances in Africa: A critical review. Sci Total Environ 739:139913

44. Sun R, Wu M, Tang L, Li J, Qian Z, Han T, Xu G (2018) Perfluorinated compounds in surface waters of Shanghai, China: Source analysis and risk assessment. Ecotoxicol Environ Saf 149:88-95

45. Thomaidi VS, Tsahouridou A, Matsoukas C, Stasinakis AS, Petreas M, Kalantzi OI (2020) Risk assessment of PFASs in drinking water using a probabilistic risk quotient methodology. Sci Total Environ 712:136485

46. UNEP (2019) Draft risk management evaluation perfluorohexane sulfonic acid (PFHxS), its salts and PFHxS-related compounds

47. http://chm.pops.int/TheConvention/POPsReviewCommittee/Meetings/POPRC15/Overview/tabid/8052/Default.aspx

48. Xie L-N, Wang X-C, Dong X-J, Su L-Q, Zhu H-J, Wang C, Zhang D-P, Liu F-Y, Hou S-S, Dong B, Shan G-Q, Zhang X, Zhu Y (2021) Concentration, spatial distribution, and health risk assessment of PFASs in serum of teenagers, tap water and soil near a Chinese fluorochemical industrial plant. Environment International 146

49. Xu C, Liu Z, Song X, Ding X, Ding D (2021) Legacy and emerging per- and polyfluoroalkyl substances (PFASs) in multi-media around a landfill in China: Implications for the usage of PFASs alternatives. Sci Total Environ 751:141767

50. Yang L, Zhu L, Liu Z (2011) Occurrence and partition of perfluorinated compounds in water and sediment from Liao River and Taihu Lake, China. Chemosphere 83, $806-14$

51. Zhang DQ, Wang M, He Q, Niu X, Liang Y (2020) Distribution of perfluoroalkyl substances (PFASs) in aquatic plant-based systems: From soil adsorption and plant uptake to effects on microbial community. Environ Pollut 257:113575

52. Zhao YG, Wan HT, Law AY, Wei X, Huang YQ, Giesy JP, Wong MH, Wong CK (2011) Risk assessment for human consumption of perfluorinated compound-contaminated freshwater and marine fish from Hong Kong and Xiamen. Chemosphere 85:277-283

53. Zhao Z, Cheng X, Hua X, Jiang B, Tian C, Tang J, Li Q, Sun H, Lin T, Liao Y, Zhang G (2020) Emerging and legacy per-and polyfluoroalkyl substances in water, sediment, and air of the Bohai Sea and its surrounding rivers. Environ Pollut 263:114391

54. Zheng P, Liu M, Yin H, Shen L, Wang Y, Wu Q (2020) Analysis of 58 poly-/perfluoroalkyl substances and their occurrence in surface water in a high-technology industrial park. Environ Pollut 267:115381

Page 10/15 
Tables

Table 1. The max and mean concentration of PFASs in water body of park A and park B (ng. L $\left.{ }^{-1}\right)$

\begin{tabular}{|c|c|c|c|c|c|c|}
\hline \multirow[t]{2}{*}{ Substances } & \multicolumn{2}{|l|}{ park $A$} & \multicolumn{2}{|l|}{ park B DS } & \multicolumn{2}{|l|}{ park B WS } \\
\hline & $\begin{array}{l}\text { Surface } \\
\text { water }(n=1)\end{array}$ & Groundwater $(n=10)$ & $\begin{array}{l}\text { Surface } \\
\text { water(n=2) }\end{array}$ & Groundwater $(n=12)$ & $\begin{array}{l}\text { Surface } \\
\text { water }(n=4)\end{array}$ & Groundwater $(n=4)$ \\
\hline F-53B & 0.35 & $0.01-0.40(0.09)$ & $\begin{array}{l}0.04- \\
0.06(0.05)\end{array}$ & $\mathrm{Nd}-0.06(0.01)$ & $0.02-0.03(0.02)$ & $\mathrm{Nd}-0.02(0.01)$ \\
\hline PFBA & 15.02 & $\begin{array}{l}1.14-2501.4 \\
(400.41)\end{array}$ & $\begin{array}{l}0.49- \\
2.29(1.39)\end{array}$ & $0.69-345.21(65.78)$ & $\begin{array}{l}48.47- \\
837.04(250.64)\end{array}$ & $\begin{array}{l}82.44- \\
527.74(289.68)\end{array}$ \\
\hline PFBS & 57.76 & $\begin{array}{l}17.07-51818.61 \\
(8734.27)\end{array}$ & $\begin{array}{l}81.20- \\
110.11(95.86)\end{array}$ & $\begin{array}{l}13.63- \\
2956.38(559.67)\end{array}$ & $\begin{array}{l}15.90- \\
3522.40(924.61)\end{array}$ & $\begin{array}{l}28.46- \\
874.23(376.10)\end{array}$ \\
\hline PFDA & 0.02 & nd-1.54(0.41) & nd-0.05(0.03) & nd-0.04(0.01) & $0.04-0.13(0.09)$ & $0.02-0.04(0.03)$ \\
\hline PFDoA & 0.00 & nd-0.62(0.08) & nd-0.03(0.01) & nd & $0.03-0.06(0.04)$ & $0.03-0.04(0.035)$ \\
\hline PFDS & 0.00 & nd-0.05(0.01) & nd & nd & nd & nd \\
\hline PFHpA & 0.84 & $1.10-681.07(94.28)$ & $\begin{array}{l}0.51- \\
0.95(0.73)\end{array}$ & nd-15.65(2.46) & $0.83-16.76(5.04)$ & $0.08-8.38(2.80)$ \\
\hline PFHXA & 1.18 & $\begin{array}{l}0.47- \\
936.32(136.09)\end{array}$ & $\begin{array}{l}0.45- \\
0.93(0.69)\end{array}$ & nd-30.943(31.80) & $\begin{array}{l}0.48- \\
97.73(25.26)\end{array}$ & $0.62-57.17(16.14)$ \\
\hline PFHxDA & 0.00 & $\mathrm{Nd}-0.42(0.06)$ & nd & nd & $0.02-0.03(0.025)$ & nd \\
\hline PFHxS & 0.00 & nd-539.60(106.87) & nd-0.13(0.07) & $0.1-9.22(0.96)$ & $0.07-1.45(0.76)$ & nd \\
\hline PFNA & 0.96 & nd-5.29(1.22) & $\begin{array}{l}0.32- \\
0.40(0.36)\end{array}$ & nd-0.23(0.02) & $0.15-0.69(0.46)$ & nd-0.07(0.035) \\
\hline PFOA & 13.46 & $\begin{array}{l}35.34- \\
11305.65(1563.13)\end{array}$ & $\begin{array}{l}9.38- \\
55.01(32.19)\end{array}$ & $\begin{array}{l}0.52- \\
1208.24(134.11)\end{array}$ & $\begin{array}{l}2.89- \\
137.93(38.42)\end{array}$ & $0.85-72.70(21.56)$ \\
\hline PFODA & 0.15 & nd- $0.75(0.16)$ & nd & nd-0.16(0.09) & $0.07-0.12(0.09)$ & $0.05-0.11(0.08)$ \\
\hline PFOS & 0.87 & $0.18-55.17(12.28)$ & $\begin{array}{l}0.56- \\
7.53(4.04)\end{array}$ & nd-29.64(2.54) & $0.05-0.62(0.32)$ & nd-0.04(0.02) \\
\hline PFPeA & 0.00 & 037-313.35(58.86) & nd-2.33(1.17) & nd-24.02(4.21) & $\begin{array}{l}0.35- \\
46.76(12.16)\end{array}$ & $0.11-29.52(8.15)$ \\
\hline PFTeDA & 0.03 & $\mathrm{Nd}-0.53(0.07)$ & nd & nd & $0.03-0.05(0.04)$ & $0.02-0.03(0.025)$ \\
\hline PFTrDA & 0.00 & $\mathrm{Nd}-0.29(0.04)$ & nd & nd & $0.06-0.11(0.08)$ & $0.04-0.05(0.045)$ \\
\hline PFUdA & 0.00 & $\mathrm{Nd}-0.54(0.07)$ & nd & nd & $0.12-0.21(0.16)$ & $0.08-0.10(0.09)$ \\
\hline
\end{tabular}

nd is not detected.

Figures 


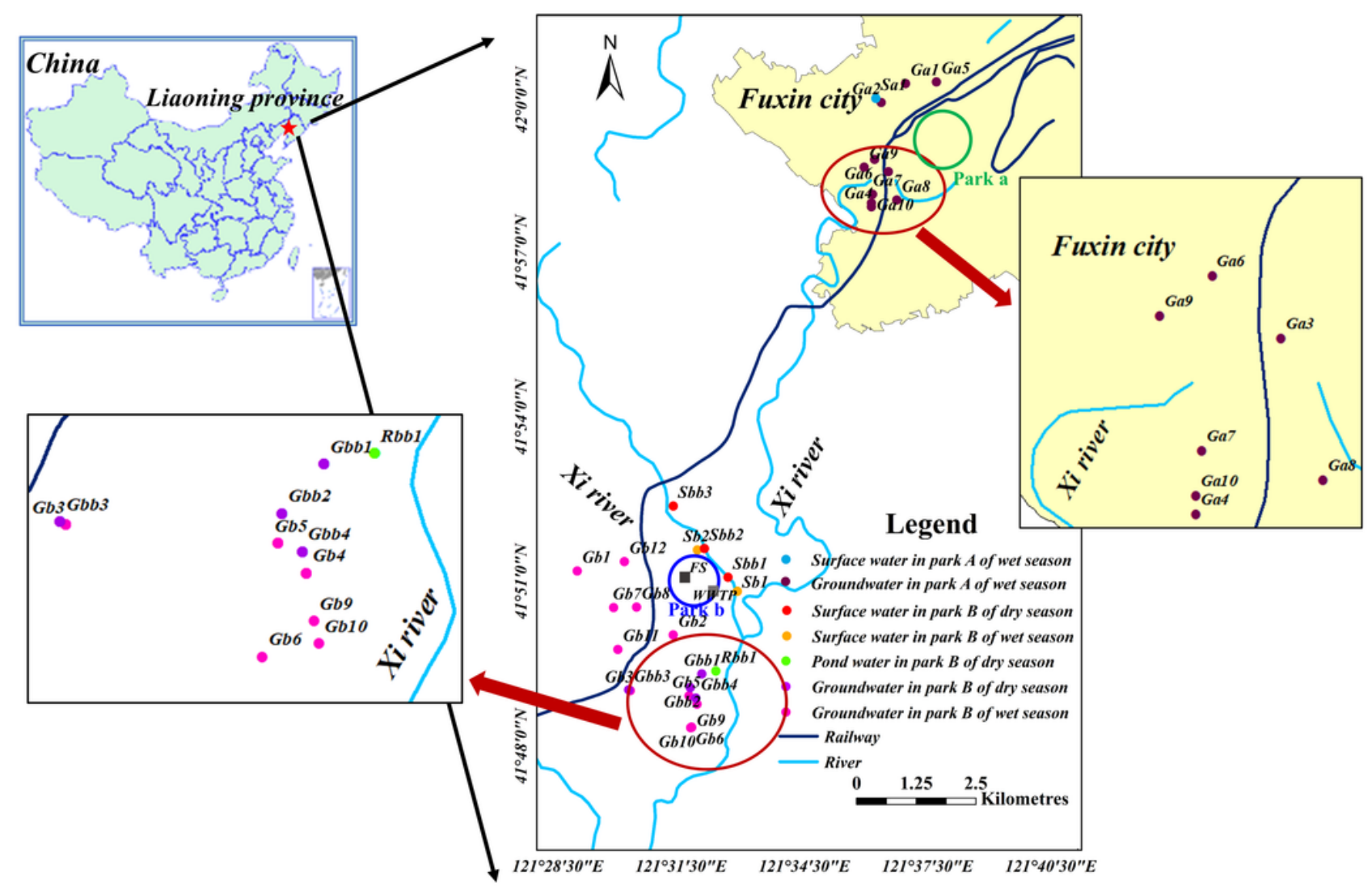

Figure 1

The sampling site of surface water and groundwater around two parks of Fuxin, China (FS is fire station and WWTP is waste water treatment plant) 
(a)

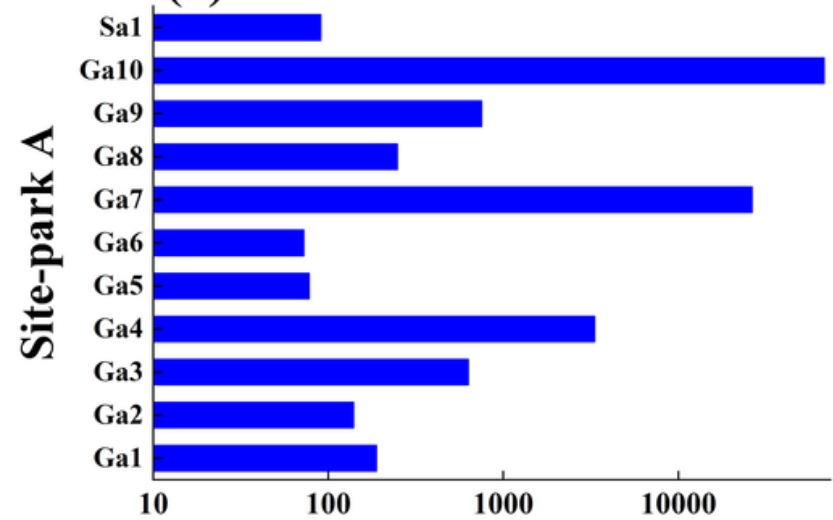

(c)

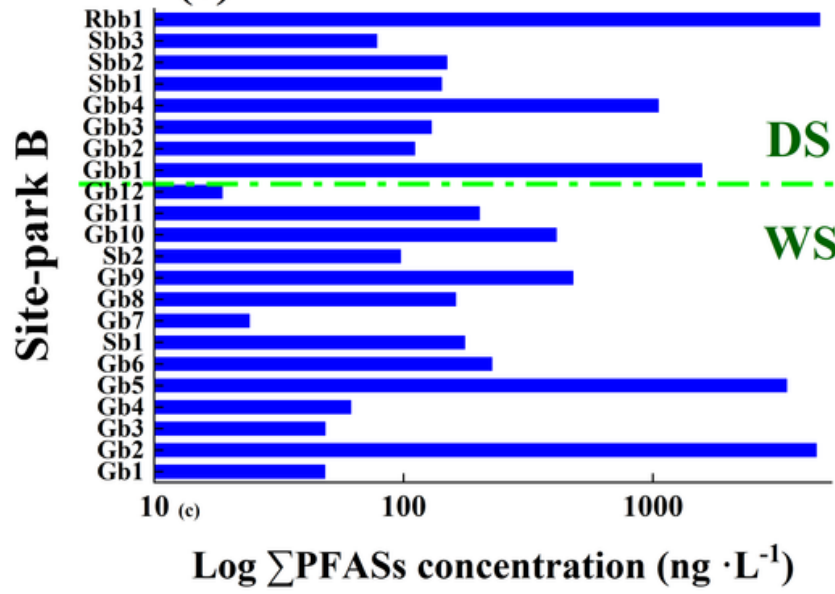

(b)

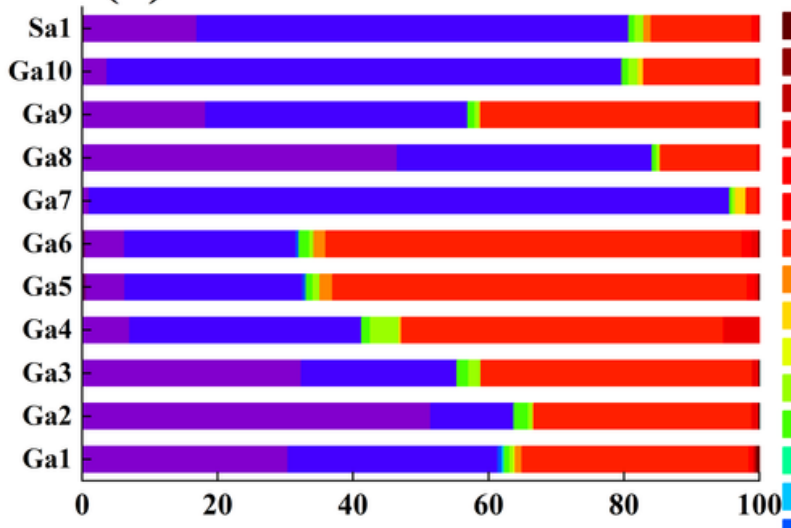

(d)

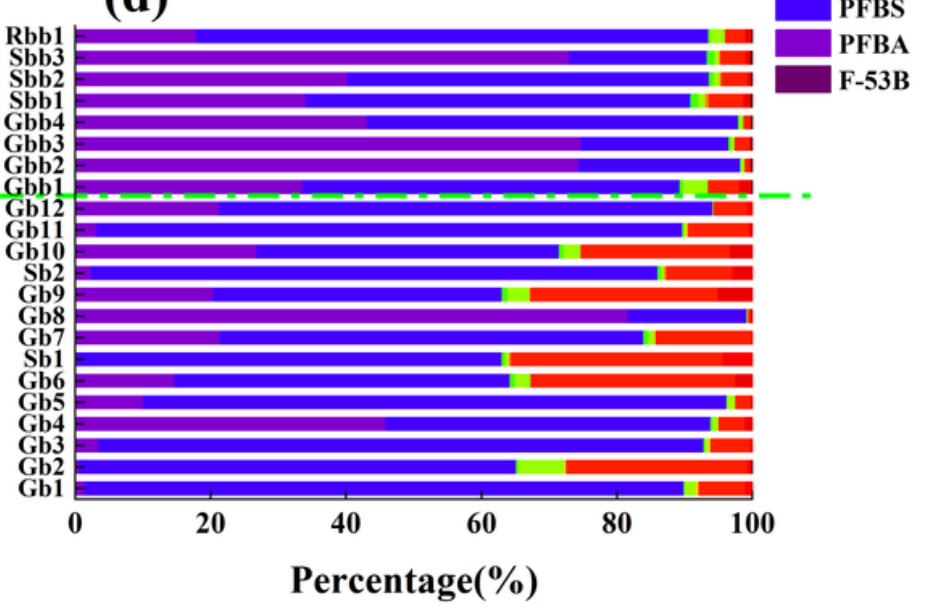

Figure 2

The concentrations of $\sum$ PFASs and profiles of 18 PFASs in park A and park B-DS and WS of Fuxin, China 


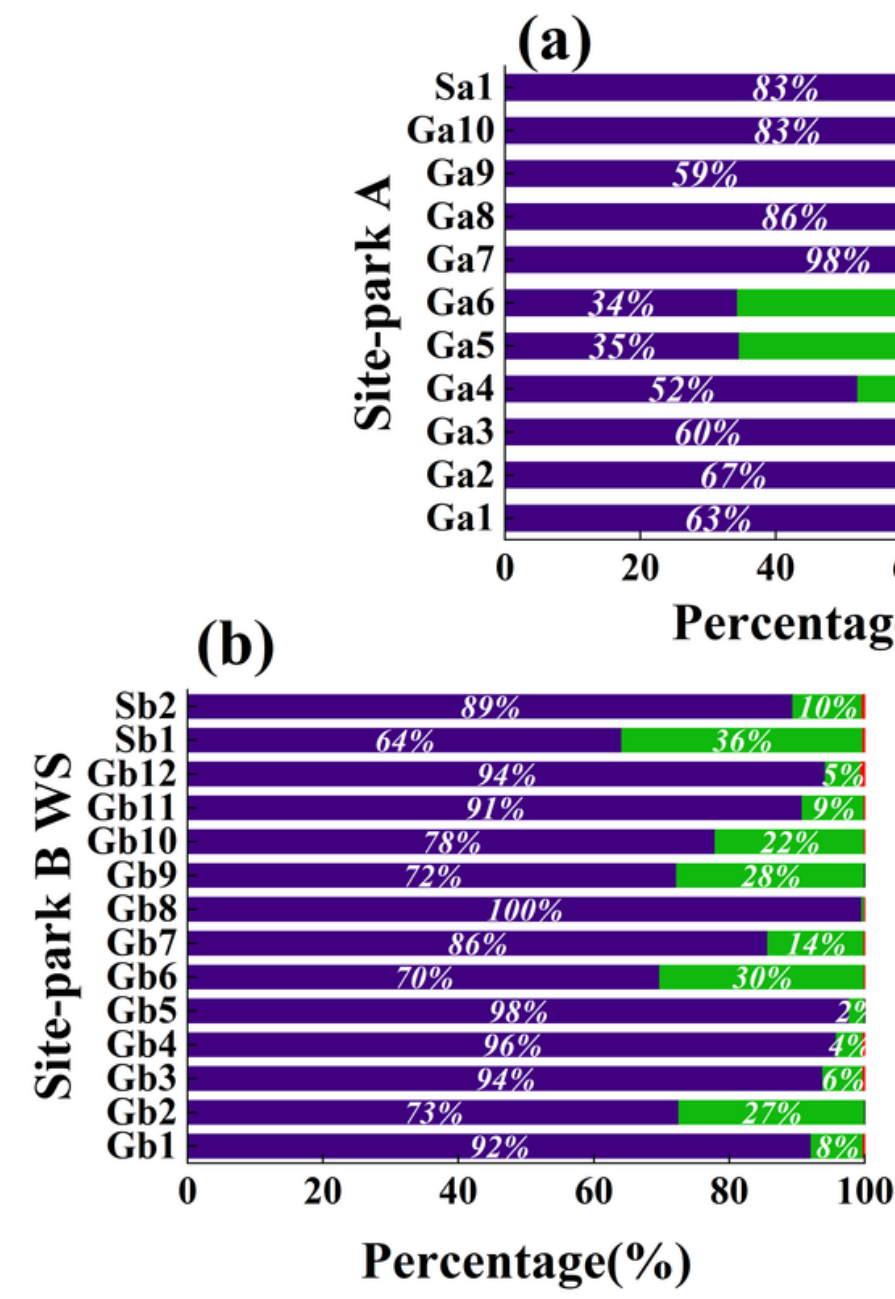

Figure 3

The concentration contribution rate (\%) basing on different carbon chain length of C4-C7 (short chain), C8 (long chain) and C9-C18 (long chain) in two parks of Fuxin, China 


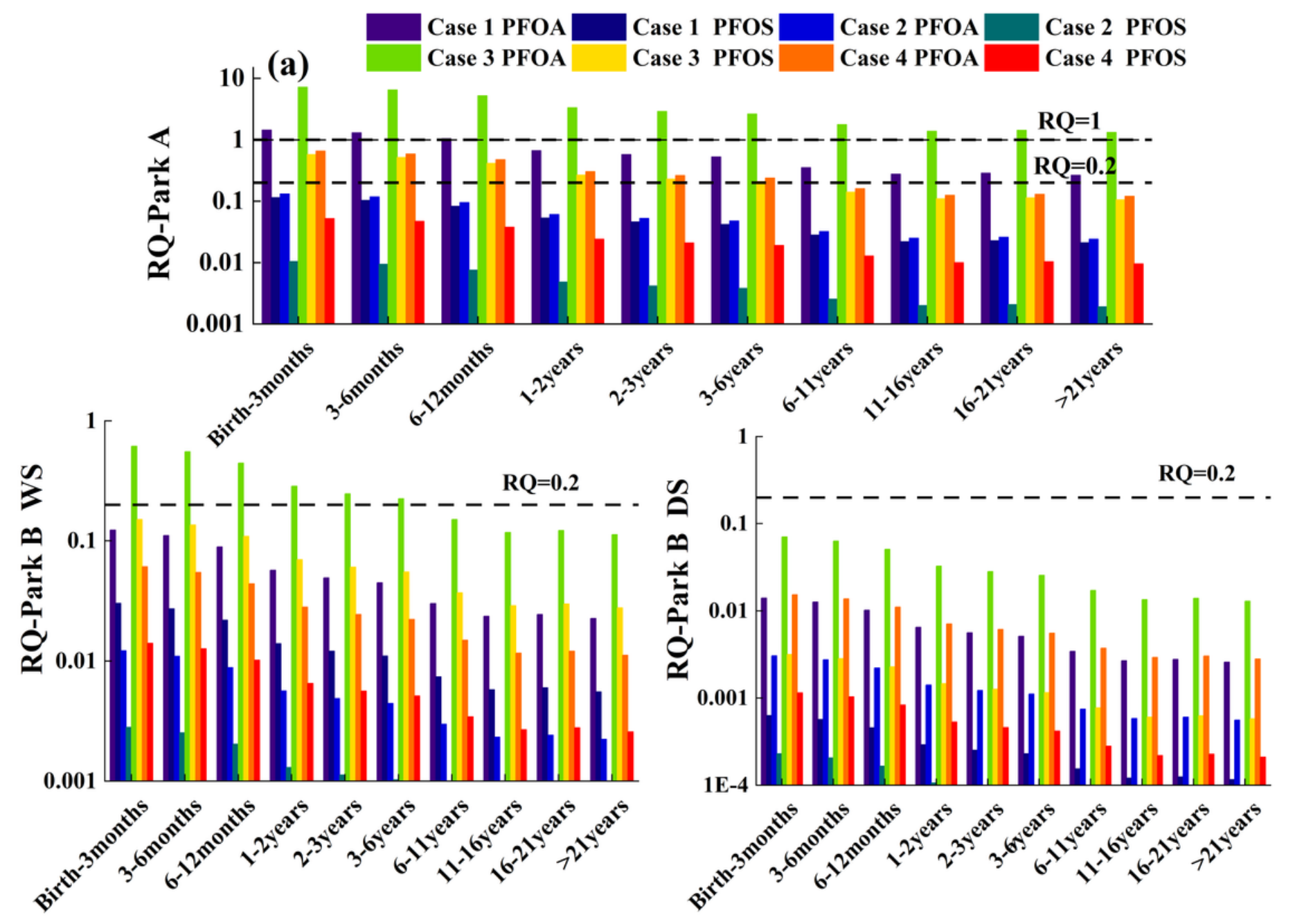

Figure 4

The RQ value of PFOA and PFOS in case 1-4 from park A and park B-WS and DS of Fuxin, China (case 1: the max concentration of RSC100\%, case 2: the mean concentration of RSC100\%, case 3: the max concentration of RSC20\% and case 4: the mean concentration of RSC20\%)

\section{Supplementary Files}

This is a list of supplementary files associated with this preprint. Click to download.

- Supportinginformation.doc 